\title{
An Approach to Reduce Part of Speech Ambiguity Using Semantically Annotated Lexicon Definitions
}

\author{
Andrei Mincă, Ştefan Diaconescu \\ Research and Development Department SOFTWIN Bucharest, Romania \\ aminca@softwin.ro,sdiaconescu@softwin.ro
}

Keywords: Lesk algorithm; knowledge-based Word Sense Disambiguation; semantically annotated glosses; lexicon network; semantic signature; semantic similarity cost; POS ambiguity

\begin{abstract}
In computational linguistics, the problem of word-sense disambiguation (WSD) is a difficult one and methods using a flat topology of the tokens are not very effective. One solution to this is to use a Part of Speech (POS) tagger before starting the WSD process. However, POS taggers show their limitations when high precision tagging is required or large texts are processed. This paper presents a technique to reduce the POS ambiguity using semantic information. As benchmarks we use as following standard WSD corpuses: Senseval2, Senseval3 and Semcor. Moreover, we tested our approach on WordNet semantically tagged glosses for English and on our own semantically tagged lexicon glosses for Romanian language.
\end{abstract}

\section{Introduction}

"All-Words" task for word sense disambiguation (WSD) is a complex pursuit in the field of natural language processing (NLP). WSD systems have improved over time and achieve now 65$70 \%$ accuracy for the fined-grained all-words task and $78-83 \%$ accuracy when a coarse-grained sense inventory is used [8].

Systems using knowledge-based methods are beginning to be the predominant research direction for WSD ([7],[9],[11]). Such knowledge-based methods have the property of low or no variation in decision making when solving sense ambiguity. Best-known results for this type of systems can go up to an accuracy of $83 \%$ on coarse-grained all-words task using an algorithm called Structural Semantic Interconnections [3].

However, obtaining a high accuracy comes at a price, i.e. large space and time consumption is needed. For tasks using fined-grained sense inventory, this problem is usually solved with heuristic approaches. Even if the POS tagging process has a lower complexity than the WSD process, its complexity is high enough to become problematic for most NLP applications. Moreover, comprehensive grammar analysis becomes difficult to accomplish [6].

Based on the above observations, we decided to investigate a partial WSD analysis before the POS tagger process. We do this in a research project in our company, codenamed SenDiS (Sense Disambiguation System).

The purpose of this paper is to significantly reduce the word sense ambiguity and thus the POS ambiguity, but still to preserve all or most of POS tagging solutions. For this purpose, we adjusted the methods and algorithms used in the SenDiS project such that the system will provide WSD variants, for a given text, with semantic similarity scores greater than a threshold relative to the maximum discovered.

\section{SenDiS WSD approach}

The SenDiS research project addresses the WSD process in a knowledge-based fashion. It mainly relies on semantic networks, especially semantic networks built from semantically annotated lexicon glosses, for establishing the sense semantic similarity costs used to solve sense ambiguity.

The main WSD usage scenario in the SenDiS system is:

1. the text is tokenized in text items;

2. each text items is matched with sense interpretations; 
3. for each sense interpretation assigned to the text items, a sense semantic signature is built based on the lexicon network;

4. relevant sense pairs with senses of different text items, are identified and then sense semantic signatures are compared;

5. semantic similarity costs of the senses in each pair are further used to compute the best WSD variant or variants.

Reference [10] describes the main ingredients of WSD approach used in the SenDiS project. This WSD approach mainly consists of the following steps detailed below:

\section{A. Lexicon nework}

A lexicon network is obtained from semantically annotated lexicon glosses [5]. This is similar to other hierarchical networks built on lexicons [4]. However, it best resembles the Lesk algorithm approach ([1],[2]) in the way that it extends the definition domain of a word sense from a set of words in a gloss to a spanning tree like structure inside this lexicon network.

Significant efforts were undertaken to achieve high quality semantic annotation of lexicon glosses. Semi-automated annotation is generally employed, but manual annotation is the gold standard even if the cost is much higher.

\section{B. Ordering the lexicon network}

The original lexicon network can be preprocessed in order to better fit different WSD methods that operate on it. This optimization task is often challenging considering the large dimensions of such networks.

\section{Building sense semantic signatures}

Using this large lexicon network, sense semantic signatures can be built having one of the forms:

- spanning tree with node (sense) and relation information embedded

- sets of nodes or/and relations

- sequences of nodes or/and relations

- combinations of the above.

\section{Comparing sense semantic signatures}

Semantic similarity cost for two senses can be obtained by comparing their semantic signatures. Various comparisons algorithms can be imagined depending on the form of the sense semantic signatures.

\section{E. Computation of WSD variants}

The final step in this WSD approach is to use these semantic similarity costs between the senses of the text items to compute best WSD variant or variants. One method will be to compute the complete sub-graph for the text, consisting of senses as nodes and edges between them with semantic similarity cost as edge rank, which maximizes the semantic similarity score of a variant.

\section{Reducing POS ambiguity using semantic information}

We propose, in analyzing a text, the computation of specific several WSD variants, especially those with strong semantic similarity scores. These should preserve the POS solutions for the text with high precision and, at the same time, should reduce the POS ambiguity for the POS tagger process.

The normal output of the SenDiS system after disambiguating a text is a WSD variant or a set of WSD variants that have the highest score of semantic similarity. We modified the last step in the system, i.e. the computation of WSD variants, in order to obtain more WSD variants with a semantic similarity score close to the highest one determined.

The last step of the SenDiS system, the computation of WSD variants with the highest semantic similarity score, has as input a set of sense pairs from the system's input text and each sense pair is associated with a semantic similarity cost. These pairs can be seen as edges in a graph where words senses are nodes. In fact this graph is actually an $\mathrm{N}$-partite graph, as seen in Figure 1, where $\mathrm{N}$ is the number of words in the text and each partition of nodes consists of nodes representing senses of the 
same word. Figure 1 has an example of a best variant highlighted. There can be more than one best variant.

The challenge is to determine the complete sub-graph, with a single node in each partition, which maximizes the sum of edges ranks.

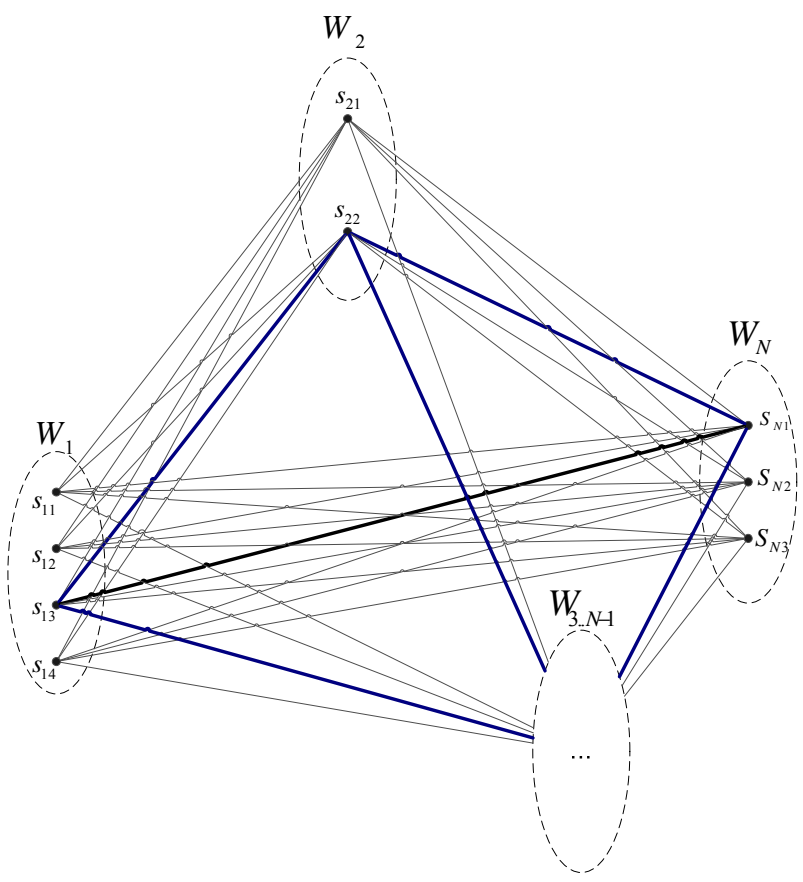

Figure 1. A graph for an N-word text

In our current implementation of this step, we first sort downward the edges and then start the construction of WSD variants, in this order, to the complete sub-graph mentioned above. Parsing the edges in this order guarantees that we will find variants with high semantic similarity score from the beginning. This order also provides a way to stop the search for variants if no other better ones are discovered. This algorithm can be seen as a dynamic backtracking for constructing high semantic similarity score WSD variants.

We propose a simple modification of this algorithm in order to obtain a set of high score variants relative to the maximum variant score determined. This is done by searching for variants until there are no more possible variants with the score greater than a threshold relative to the best variant possible and already discovered. Also, at the end of the search, this threshold is used to remove the variants constructed so far not above the threshold.

We observe a trend in the discovering of the WSD variants when using this approach. It usually starts with high similarity scores and rapidly reaches the maximum similarity score that a WSD variant can have for the entry text, after which it drops very fast. However, in ambiguous texts or in texts with strong semantic similarities between word senses the falling of the trend may occur much slower or later in the search for variants with score above a given threshold relative to the highest variant possible.

\section{Results}

To test our assumptions, we applied our methods on the English and Romanian languages. For English, we used the WordNet semantically annotated glosses as a lexicon network and Senseval2, Senseval3, Semcor and WordNet glosses as WSD corpuses. As for Romanian, we used our own lexicon with semantically annotated glosses and the same as WSD corpus.

\section{A. English tests}

Since WordNet is organized in so-called synsets, we modified this structure to apply our methods. This translates in multiplying the gloss text for every term in the synset. 
TABLE I. WORDNET AS A LEXICON STRUCTURE

\begin{tabular}{|c|c|c|c|}
\hline \multicolumn{4}{|c|}{ Lexicon structure } \\
\hline Synset terms & Senses & Gloss tokens & Annotated gloss tokens \\
\hline 155,287 & 206,941 & $3,114,968$ & 936,397 \\
\hline
\end{tabular}

TABLE II. WORDNET LEXICON NETWORK

\begin{tabular}{|c|c|}
\hline \multicolumn{2}{|c|}{ Lexicon network } \\
\hline Senses as nodes & Sense relations \\
\hline 206,941 & 936,397 \\
\hline
\end{tabular}

The following table presents the probability of the POS solution to be present in the reduced POS ambiguity.

TABLE III. ENGLISH PROBABILITY OF POS SOLUTION IN POS REDUCED AMBIGUITY

\begin{tabular}{|c|c|c|c|c|}
\hline \multicolumn{5}{|c|}{ PROBABILITY OF POS SOLUTIONS IN POS REDUCED AMBIGUITY } \\
\hline Sentences & WSD Corpus & $\boldsymbol{P}$ & $\boldsymbol{R}$ & F measure \\
\hline 238 & Senseval2 & 0.9098 & 0.8531 & 0.8782 \\
\hline 300 & Senseval3 & 0.9171 & 0.8747 & 0.8930 \\
\hline 37,176 & Semcor & 0.9136 & 0.8659 & 0.8855 \\
\hline 165,977 & WordNet glosses & 0.9022 & 0.8866 & 0.8930 \\
\hline
\end{tabular}

The following graphics show the logarithmic scale representing the degree of POS ambiguity reduction (computed as reduced ambiguity over initial ambiguity) along the number of items in the text, for each English corpus used for testing.

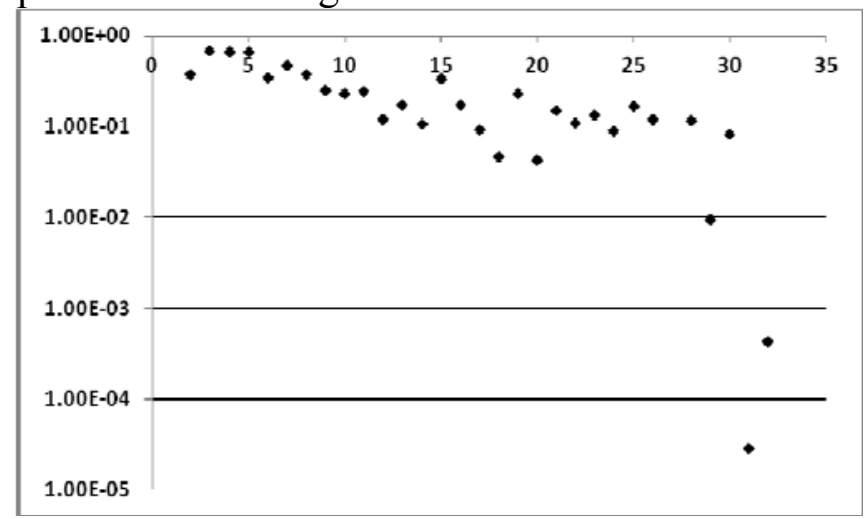

Figure 2. Senseval2 Log scale of POS ambiguity reduction degree

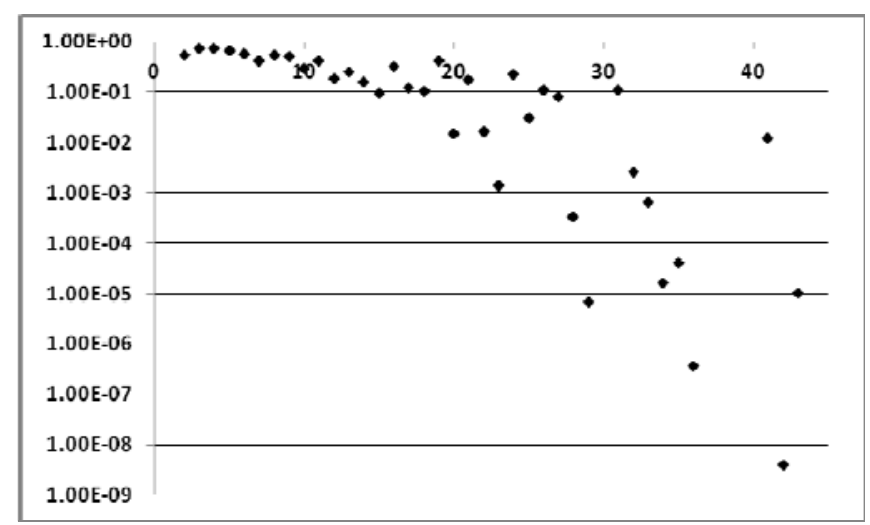

Figure 3. Senseval3 Log scale of POS ambiguity reduction degree 


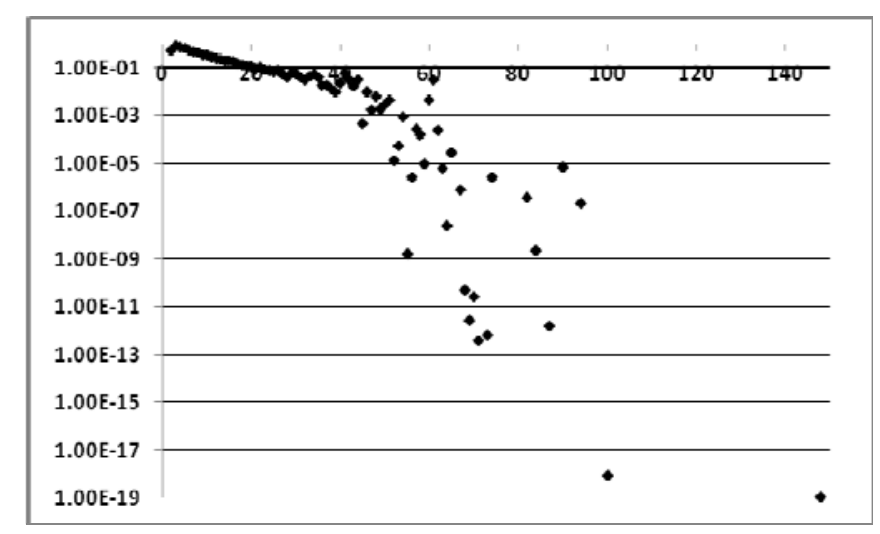

Figure 4. Semcor Log scale of POS ambiguity reduction degree

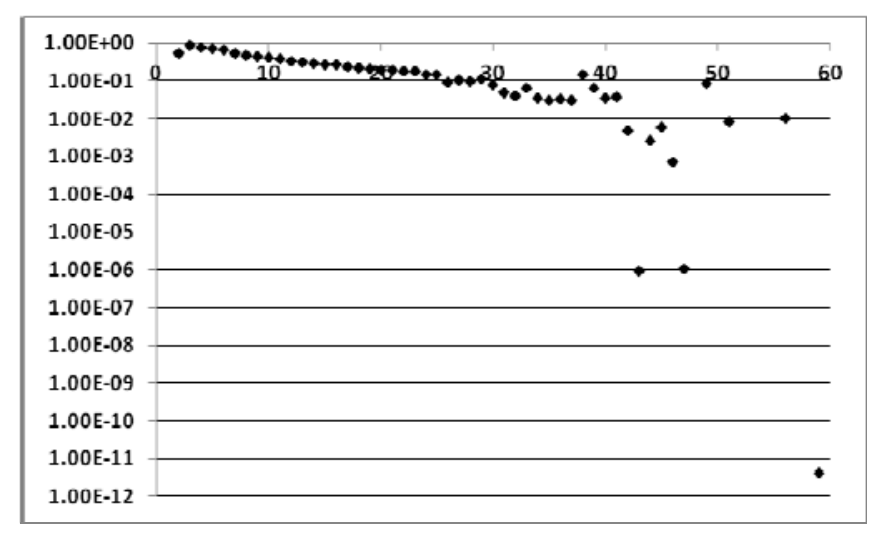

Figure 5. WordNet glosses Log scale of POS ambiguity reduction degree

The following figures show the trend for WSD variants discovering. The Y-axis represents the similarity scores mapped to a $0-100 \%$ interval where $100 \%$ is the highest score and $0 \%$ is the lowest score for similarity. The X-axis represents the WSD variants indexes as they are discovered by our WSD solution, also mapped to a $0-100 \%$ interval. The threshold for the similarity score was set to $90 \%$.

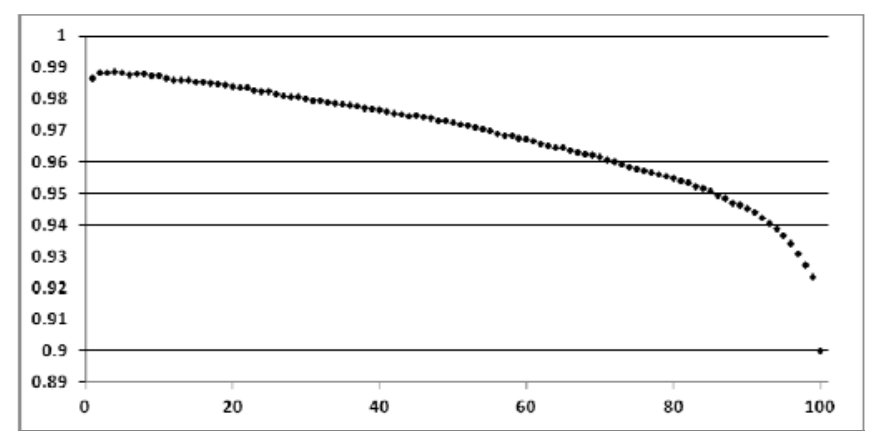

Figure 6. Senseval2 trend for WSD variants score

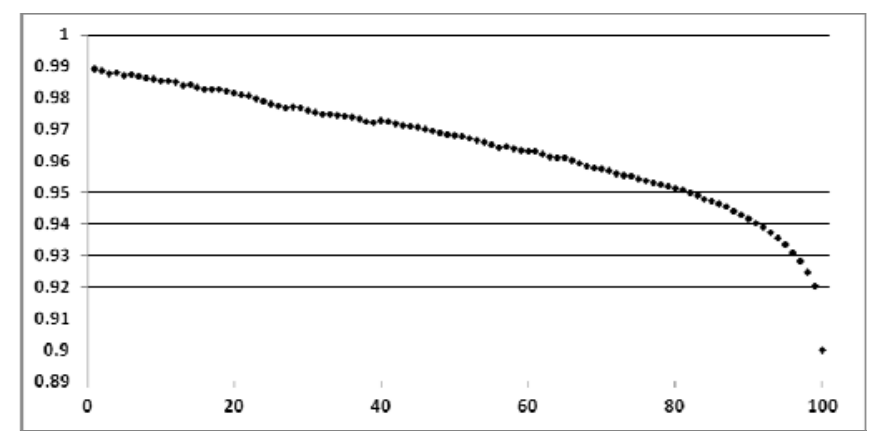

Figure 7. Senseval3 trend for WSD variants score 


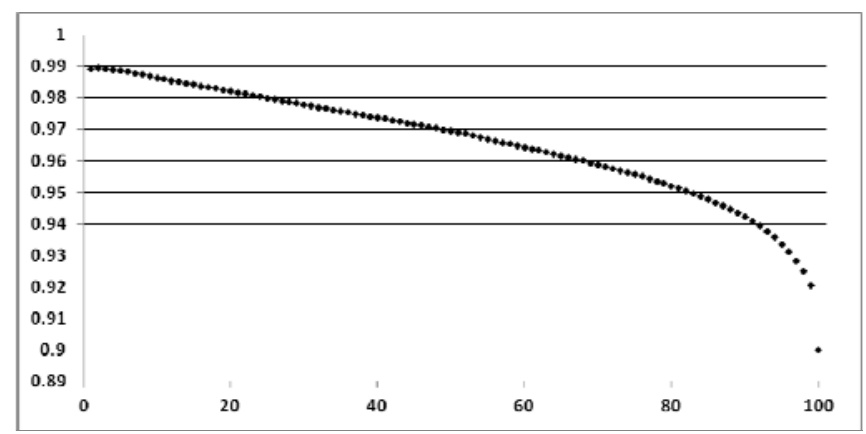

Figure 8. Semcor trend for WSD variants score

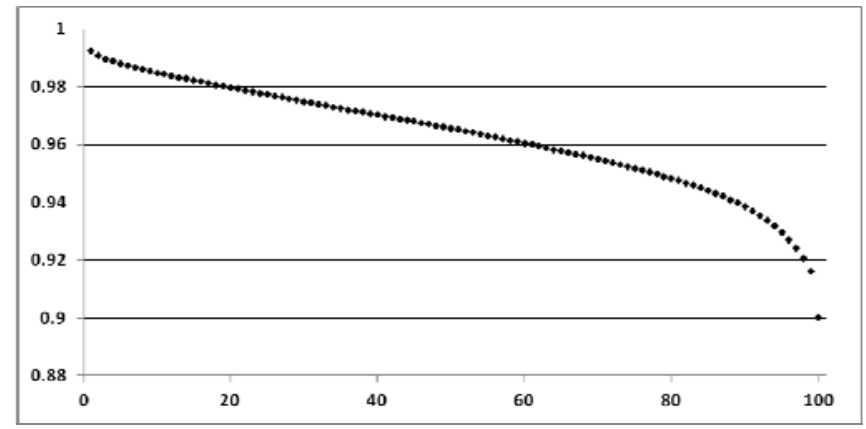

Figure 9. WordNet glosses trend for WSD variants score

\section{B. Romanian tests}

For our Romanian lexicon we added a cost of context relevance in the manual annotation process. There are three values for context relevance, assigned to a text item, in the form of Weak, Medium, and Strong.

TABLE IV. ROMANIAN LEXICON

\begin{tabular}{|c|c|c|c|}
\hline \multicolumn{4}{|c|}{ Lexicon structure } \\
\hline Lexicon entries & Senses & Gloss tokens & Annotated gloss tokens \\
\hline 90,012 & 130,099 & $1,528,991$ & 686,787 \\
\hline
\end{tabular}

TABLE V. ROMANIAN LEXICON NETWORK

\begin{tabular}{|c|c|c|c|}
\hline \multicolumn{4}{|c|}{ Lexicon network } \\
\hline Senses & Weak relations & Medium relations & Strong relations \\
\hline 120,514 & 177,404 & 210,081 & 299,302 \\
\hline
\end{tabular}

TABLE VI. ROMANIAN PROBABILITY OF POS SOLUTION IN POS REDUCED AMBIGUITY

\begin{tabular}{|c|c|c|c|}
\hline \multicolumn{4}{|c|}{ PROBABILITY OF POS SOLUTIONS IN POS REDUCED } \\
AMBIGUITY
\end{tabular}

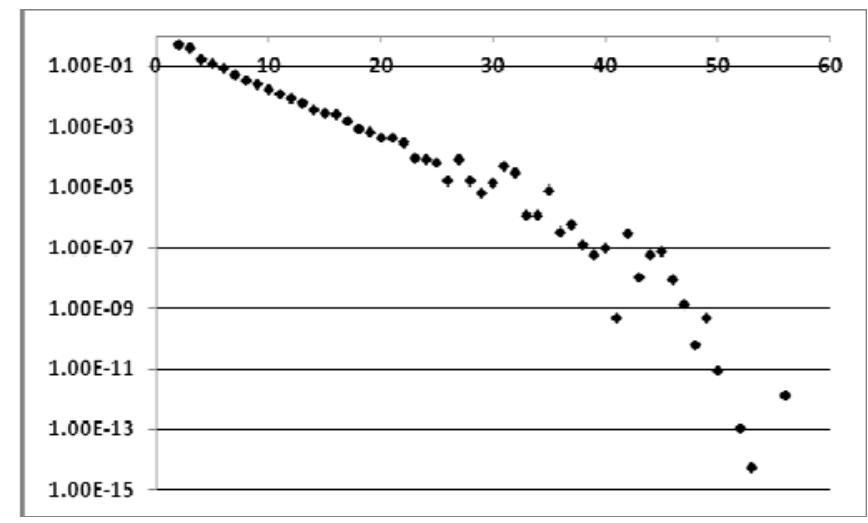

Figure 10. Romanian lexicon Log scale of POS ambiguity reduction degree 


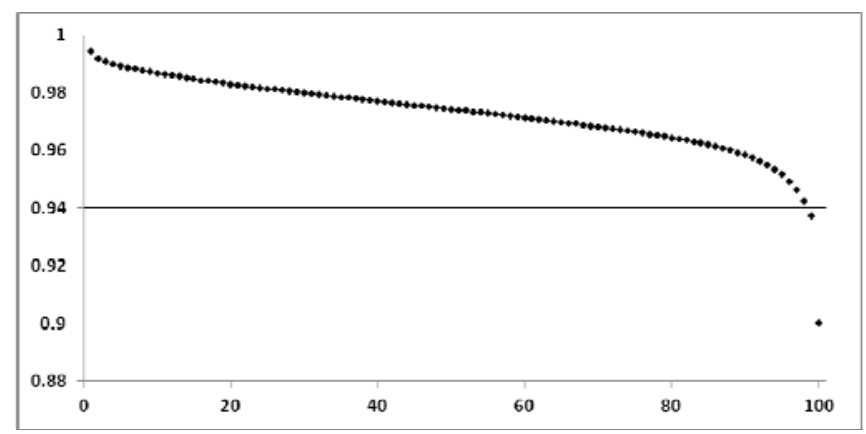

Figure 11. Romanian lexicon glosses trend for WSD variants score

\section{Conclusions}

We presented a method for POS ambiguity reduction with a high probability for solutions preservation, using a WSD method that provides WSD variants within a threshold of $90 \%$ relative to the highest possible WSD variant score. As we show in the article, our method has around 90\% accuracy of POS solution preservation with a reduction of POS ambiguity. In average, POS ambiguity is reduced to below $20 \%$ when applying our method.

Moreover, the results were even higher for Romanian language because our lexicon has more inflection form entries that improve the finding of senses for text items.

Our research results open a new way of approaching comprehensive grammar analysis, by using them for reducing the complexity of the text.

\section{Acknowledgment}

The research in this paper was carried out in the project "General Word Sense Disambiguation System applied to Romanian and English Languages" (SenDiS), co-funded by a Romanian research grant no. 207/20.07.2010 from the "Research, Technological Development and Innovation for Competitiveness" program.

\section{References}

[1] M. Lesk, "Automatic sense disambiguation using machine readable dictionaries: How to tell a pine cone from an ice cream cone," Proceedings of SIGDOC '86, 1986.

[2] S. Banerjee and T. Pedersen, "An adapted Lesk algorithm for word sense disambiguation using WordNet," Proc. of the Third International Conference on Intelligent Text Processing and Computational Linguistics, Mexico City, 2002.

[3] R. Navigli and P. Velardi, "Structural semantic interconnections: a knowledge-based approach to word sense disambiguation," IEEE Transactions Pattern Analysis and Machine Intelligence, vol. 27, issue 7, pp. 1075-1086, 2005

[4] WordNet at Princeton University, http://wordnet.princeton.edu

[5] Semantically Tagged glosses for WordNet, http://wordnet.princeton.edu/glosstag.shtml.

[6] Şt. Diaconescu,"Natural Language Syntax Description using Generative Dependency Grammar," POLIBITS 38, 5-18, July-December 2008, ISSN: 1870-9044

[7] R. Navigli, "Using Cycles and Quasi-Cycles to Disambiguate Dictionary Glosses", EACL '09 Proc. of the 12th Conference of the European Chapter of the Association for Computational Linguistics, Stroudsburg, PA, USA, March 2009.

[8] R. Navigli, "Word sense disambiguation: A survey," ACM Comput. Surv. 41, 2, Article 10, February 2009.

[9] S. P. Ponzetto and R. Navigli, "Knowledge-rich Word Sense Disambiguation rivaling supervised systems," Proc. of the 48th Annual Meeting of the Association for Computational Linguistics (ACL), pp. 1522-1531, 2010. 
[10] A. Minca and St. Diaconescu, "An approach to knowledge-based Word Sense Disambiguation using semantic trees built on a WordNet lexicon network," Proc. of the 6th International Conference on Speech Technology and Human-Computer Dialogue (SpeD 2011), pp. 1-6, Brasov, Romania (May, 2011)

[11] Y. Gutiérrez, Sonia Vázquez and A. Montoyo, "A graph-based approach to WSD using relevant semantic trees and n-cliques model," Proc. of the 13th International Conference on Computational Linguistics and Intelligent Text Processing (CICLing 2012), vol. Part I, pp. 225-237, 2012 